\title{
Evaluation of Dicationic Reagents for Their Use in Detection of Anions Using Positive Ion Mode ESI-MS Via Gas Phase Ion Association
}

\author{
Jeffrey W. Remsburg, Renee J. Soukup-Hein, Jeffrey A. Crank, \\ Zachary S. Breitbach, Tharanga Payagala, and Daniel W. Armstrong \\ Department of Chemistry and Biochemistry, University of Texas at Arlington, Arlington, Texas, USA
}

Twenty-three different dications were investigated for their effectiveness in pairing with singly charged anions, thereby allowing the electrospray ionization mass spectrometry (ESI-MS) detection of anions as positively charged complexes. Nitrate, iodide, cyanate, monochloroacetate, benzenesulfonate, and perfluoro-octanoate were chosen as representative test anions as they differ in mass, size-to-charge ratio, chaotropic nature, and overall complexity. Detection limits were found using direct injection of the anion into a carrier liquid containing the dication. Detection limits are given for all six anions with each of the 23 dications. Each anion was easily detected at the $\mathrm{ppb}(\mu \mathrm{g} / \mathrm{L})$ and often the ppt $(\mathrm{ng} / \mathrm{L})$ levels using certain dicationic reagents. The ability of dicationic reagents to pair with anions and produce ESI-MS signals varied tremendously. Indeed, only a few dications can be considered broadly useful and able to produce sensitive results. Liquid chromatography (LC)-ESI-MS also was investigated and used to show how varying the dicationic reagent produced significantly different peak intensities. Also, the use of tandem mass spectrometry can lead to even greater sensitivity when using imidazolium based dications. (J Am Soc Mass Spectrom 2008, 19, 261-269) () 2008 American Society for Mass Spectrometry

$\mathrm{D}$ etection and quantitation of anions is important in a wide variety of scientific fields. Scientists in environmental chemistry, biochemistry, and the food and drug industries all routinely use analytical techniques to study anions. The most common methods for anion analysis include ion-selective electrodes [1, 2], ion chromatography (IC) [3, 4], flow injection analysis (FIA) [5, 6], and a variety of other spectroscopic and electroanalytical approaches. Mass spectrometry is an obvious choice for detection of anions since they are charged species. The advent of electrospray ionization allowed routine analysis of the ionic components in a liquid sample [7]. By coupling ESI-MS with a separation method (i.e., liquid chromatography), a means to separate and detect most compounds is easily accomplished. However, while ESI-MS is widely used in both the positive and negative ion modes, the positive ion mode often is preferred as it can have lower detection limits and higher stability [8-10]. For positive mode analysis, an acidic additive is commonly employed to facilitate protonation of the analyte and to provide a stable electrospray. However, the addition of a basic compound to a water/methanol sol-

Address reprint requests to Dr. Daniel W. Armstrong, Department of Chemistry and Biochemistry, The University of Texas at Arlington, Arlington, TX 76019, USA. E-mail: sec4dwa@uta.edu vent system does not seem to provide a stable spray for negative mode analysis, resulting in fluctuations of the ion current [8]. It is known that corona discharge is more prevalent in the negative ion mode as opposed to the positive ion mode, which can produce a significant rise in background peaks and can also lead to reduced stability for the ion current [11]. Also, undesirable arcing is more prevalent in the negative ion mode. It has been suggested that halogenated solvents such as chloroform [12], hexafluoroisopropanol [13], and 2,2,2,-trifluororethanol [8] be used as opposed to more common solvents. These halogenated solvents produce an abundance of halogen ions at the capillary tip, resulting in a more stable spray formation. To reduce the occurrence of corona discharge, both electron-scavenging gases [14] and halogenated solvents [15] have proven useful. While carefully choosing amongst the aforementioned solvents may lead to better signals in the negative ion mode, it must be noted that these are not common solvents for use in LC, IC, or FIA. Ideally, one would like to be able to use common solvents such as methanol and water and also take advantage of using the positive ion mode, so less optimization is necessary and the problems with negative mode can be avoided.

Recently a method was developed to detect singly charged anions in the positive ion mode, thus eliminat- 
ing the necessity of using negative ion mode and also eliminating any need for unconventional solvents. This method entails the addition of a small amount of a relatively large, chaotropic, organic dication to the carrier flow solvent, which can pair with a single anion to give a positively charged complex of a higher $\mathrm{m} / \mathrm{z}$. This approach was first used for the trace analysis of perchlorate [16-19]. Most recently, it was shown to be advantageous for the analysis of over 30 different anions, proving its broad applicability and effectiveness [20]. There are several advantages to this method, among the more important of which are its ease of use and its sensitivity. Indeed, this single method provided the best reported limits of detection (LOD) for a variety of anions, proving to be more sensitive than negative ion mode ESI-MS methods as well as other analytical techniques [20]. Only a small concentration of a dication reagent is needed (tens of $\mu \mathrm{M}$ ) and it can be added post-column if a separation method is employed so there is no effect on the separation. Finally, there is a key advantage to this method when it is employed with certain quadrupole instruments. By pairing the anion with a large dication, one can eliminate any problems with detection of an anion either below or near the low mass cutoff (LMCO). That is, whereas the anion previously may either have fallen below the LMCO or so close to it that detection was severely hindered, it can be paired with a dication, thereby moving the detected mass-to-charge ratio several hundred units higher, to a region of low background interference noise and few interfering peaks.

Apart from the original perchlorate study [16], there has not been any substantial amount of research done on what types of dications provide good or poor results. The dication that was found to provide the best results in the original study (1,1'-(nonane-1,9-diyl) bis(3methylimidazolium), dication VIII in Table 1 was consequently used for multi-anion study of reference [20]. Obviously, differences in the structure and nature of the dication could cause a significant difference in its affinity for different anions, as well as its stability and overall efficacy. The purpose of this study is to examine the effects of using a variety of different types and structures of dication reagents, and to determine whether or not their selectivity, sensitivity, and efficacy vary for different anions. Our previous efforts included extensive research in developing dicationic compounds [21-24]. Originally synthesized as ultra-stable ionic liquids [21], this research has led to the development of a wide array of dicationic compounds, including imidazolium [21, 22] and phosphonium unpublished results based dications, as well as those with differing linkage chains [22, 23] and even unsymmetrical species [23].

In this work, 23 dication salts are studied for their ability to form a complex with several different anions to be detected by ESI-MS. The salts encompass a wide range of cationic moieties (including imidazolium, pyrrolidinium, pyridinium and phosphonium-based cations), and structures (differing chain lengths, aromatic- ity, symmetrical and unsymmetrical dications, etc.). Detection limits via direct injection are used to determine efficacy for the complex formed between the dication (dissolved in the carrier stream) and the anion of interest. The results are evaluated to discern which reagents provide the highest selectivity and sensitivity, as well as the structural features that make an effective or ineffective pairing agent. Finally, representative LCESI-MS analyses are done to illustrate the effect of using different dicationic reagents for anion analysis in the positive ion mode.

\section{Experimental}

Methanol and water were of HPLC grade and obtained from Burdick and Jackson (Morristown, NJ). Reagent grade sodium hydroxide and sodium fluoride were from Fisher Scientific (Pittsburgh, PA). Anions used were purchased as either the sodium/potassium salt or as the free acid from Sigma-Aldrich (St. Louis, MO). Stock solutions of each anion were made weekly. Chemicals used for the syntheses of the dicationic compounds were also obtained from Sigma-Aldrich.

Dication I from Table 1 was synthesized by dissolving one molar equivalent of 1,5-dibromopropane in isopropanol. To this solution, $3 \mathrm{M}$ equivalents of tripropylphosphine were added. The resulting mixture was stirred and heated to reflux for $48 \mathrm{~h}$. The solution was then cooled to room temperature and the solvent was removed by rotoevaporation. The crude product was then dissolved in deionized water and washed several times with ethyl acetate to remove any residual starting material. The water was then removed through rotoevaporation, followed by overnight drying in vacuum over phosphorous pentoxide. Dications II, III, V-X, and XII-XVIII were made in an analogous manner. Dications $\mathbf{X I X}$ and XX were synthesized by refluxing $1 \mathrm{M}$ equivalent of (5-bromopentyl)-trimethylammonium bromide in isopropyl alcohol with $3 \mathrm{M}$ equivalents of 1-methylimidazole and tripropylphosphine, respectively. The resulting product was then purified as described above. To produce dications IV and XI, synthesis of the dibromopolyethylene glycol linker chain was first needed. This was accomplished by dissolving tetra(ethylene glycol) in ether, which was then cooled in an ice bath and reacted with $1.1 \mathrm{M}$ equivalents of phosphorus tribromide. The reaction was then refluxed for $2 \mathrm{~h}$. Next, the reaction mixture was poured over ice to react the excess $\mathrm{PBr}_{3}$. The aqueous layer was discarded and the organic layer was washed four times with an aqueous sodium bicarbonate solution. The organic layer was then dried with sodium sulfate and filtered. Next, the ether was removed by rotary evaporator and the resulting linker was placed under vacuum overnight to ensure complete dryness. This linker was then reacted with the appropriate end groups to produce the dication. Dication XXI was synthesized by first dissolving one molar equivalent 
Table 1. Structures and masses of the dications used in this study

\begin{tabular}{|c|c|c|c|c|c|}
\hline No. & Mass & Structure & No. & Mass & Structure \\
\hline I & 362.6 & & $X V$ & 228.1 & \\
\hline II & 390.6 & & $X \mathrm{VI}$ & 324.6 & \\
\hline III & 446.6 & & XVII & 188.4 & \\
\hline IV & 480.6 & & XVIII & 286.6 & \\
\hline V & 424.8 & & XIX & 211.2 & \\
\hline VI & 206.3 & & $x x$ & 289.4 & \\
\hline VII & 234.3 & & $X X I$ & 324.4 & \\
\hline
\end{tabular}


Table 1. Continued

\begin{tabular}{|c|c|c|c|c|c|}
\hline No. & Mass & Structure & No. & Mass & Structure \\
\hline VIII & 290.3 & & XXII & 184.1 & \\
\hline IX & 268.3 & & XXIII & 610.6 & \\
\hline$x$ & 420.4 & & & & \\
\hline$X I$ & 384.4 & & & & \\
\hline XII & 294.3 & & & & \\
\hline XIII & 318.4 & & & & \\
\hline XIV & 386.3 & & & & \\
\hline
\end{tabular}

of cinchonidine in $\mathrm{N}, \mathrm{N}$-dimethylformamide at $80^{\circ} \mathrm{C}$. Four molar equivalents of methyl iodide were then added to the mixture and allowed to react for $48 \mathrm{~h}$. After the solvent was removed by rotary evaporation, the residue was dissolved in methanol. Upon addition of diethyl ether, the product precipitated out of solution, and was collected by filtration and then washed with cold ether. Dications XXII and XXIII are commercially available compounds (Sigma-Aldrich). All dicationic compounds were anion exchanged to their fluoride form to maximize complex formation between the dication and the injected analyte. This anion exchange procedure is given in reference [16].

For direct injection analysis, a $40 \mu \mathrm{M}$ dication-fluoride $\left(\mathrm{DF}_{2}\right)$ solution was directed into a Y-type mixing tee at $100 \mu \mathrm{L} / \mathrm{min}$ via a Shimadzu LC-6A pump (Shi$\operatorname{madzu}$, Columbia, MD). Also directed into the mixing tee was a carrier flow consisting of a 2:1 ratio of methanol to water at $300 \mu \mathrm{L} / \mathrm{min}$ from a Surveyor MS pump (Thermo Fisher Scientific, San Jose, CA). After the mixing tee, the final conditions were then 50/50 water/methanol with $10 \mu \mathrm{M} \mathrm{DF}_{2}$ at a flow rate of 400 $\mu \mathrm{L} / \mathrm{min}$. Sample introduction was done with the six- port injection valve on the mass spectrometer using a 2 $\mu \mathrm{L}$ sample loop. A linear ion trap mass spectrometer (LXQ; Thermo Fisher Scientific, San Jose, CA) was used for this study. The ESI-MS settings were spray voltage, $3 \mathrm{kV}$; capillary temperature, $350{ }^{\circ} \mathrm{C}$; capillary voltage, $11 \mathrm{~V}$; tube lens voltage, $105 \mathrm{~V}$; sheath gas, 37 arbitrary units (AU); and auxiliary gas, 6 AU. For the negative ion mode analysis, voltage polarities were reversed, while all other parameter settings were kept. ESI-MS settings for the optimized MCA detection are as follows; spray voltage: $4.5 \mathrm{kV}$, capillary temperature: $350{ }^{\circ} \mathrm{C}$, capillary voltage: $35 \mathrm{~V}$, tube lens voltage: $80 \mathrm{~V}$, sheath gas: $25 \mathrm{AU}$, and auxiliary gas: 16 AU. The ion trap was operated using single ion monitoring (SIM).

For the chromatographic experiments, sample introduction was done by a Thermo Fisher Surveyor autosampler (10 $\mu \mathrm{L}$ injections). The stationary phase used was a $10 \mathrm{~cm}$ C-18 (3 $\mu \mathrm{m}$ particle size) obtained from Advanced Separations Technology (Whippany, NJ). In the chromatograph of the multi-anion sample used for Figure 1, the column was equilibrated with $100 \%$ water at $300 \mu \mathrm{L} / \mathrm{min}$. At $1 \mathrm{~min}$, a linear gradient to $100 \%$ 

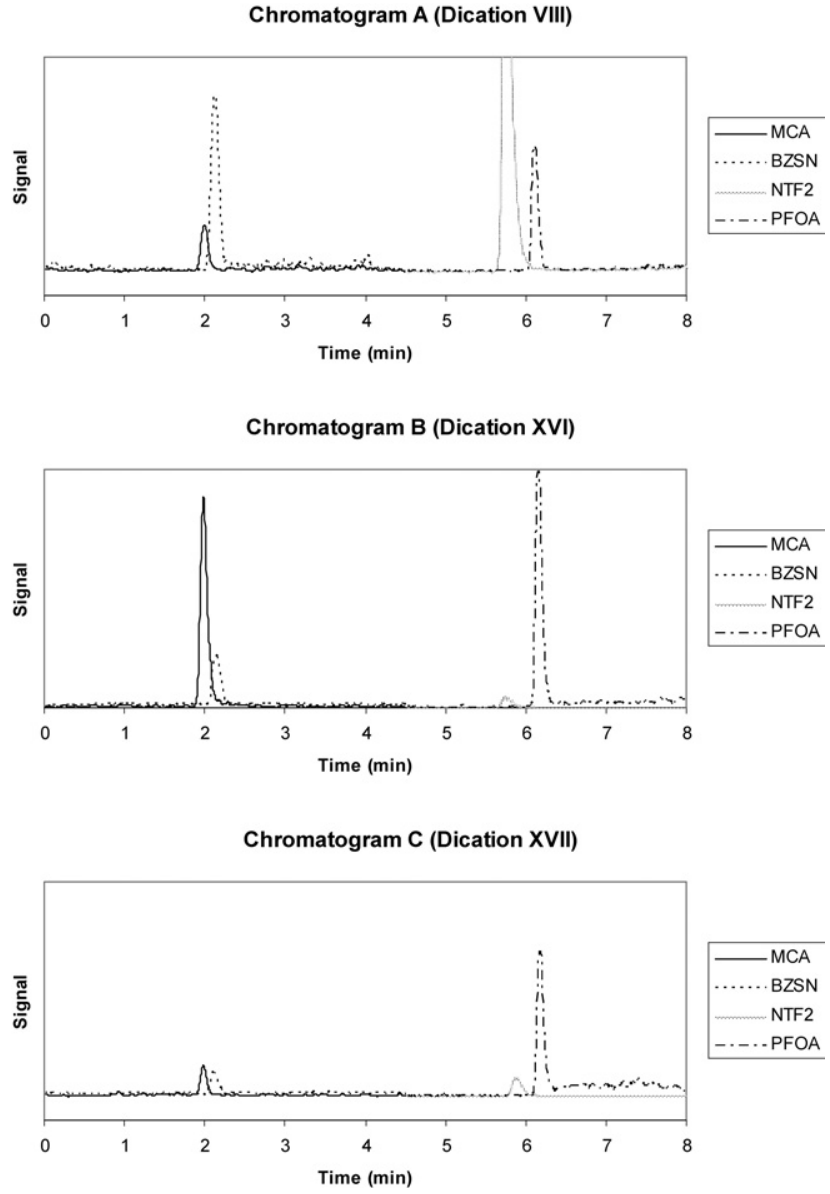

Figure 1. Three separate chromatograms showing the separation of a sample containing four anions $(150 \mathrm{ng} / \mathrm{mL} \mathrm{MCA}, 50 \mathrm{ng} / \mathrm{mL}$ BZSN, $500 \mathrm{ng} / \mathrm{mL} \mathrm{NTF}_{2}$, and $75 \mathrm{ng} / \mathrm{mL}$ PFOA). The masses monitored are the sum of the mass of each anion and the mass of the corresponding dicationic reagent. Chromatograms A and B use recommended dications (VIII and XVI), while chromatogram C does not (XVII).

methanol began and was completed at $3 \mathrm{~min}$. The addition of the $\mathrm{DF}_{2}$ solution was done post-column at $100 \mu \mathrm{L} / \mathrm{min}$ via the mixing tee. For the chromatographs of the benzenesulfonate samples, the mobile phase consisted of $100 \%$ water at $300 \mu \mathrm{L} / \mathrm{min}$ for the entire analysis. To help with spray formation, the $\mathrm{DF}_{2}$ was prepared as a methanol solution and again added post-column. For the negative ion mode runs, pure methanol was introduced into the mixing tee as opposed to the $\mathrm{DF}_{2}$ in methanol solution. The MS was again operated in SIM mode, monitoring the mass-tocharge ratio of each analyte for the entire run. Where single reaction monitoring was used, the normalized collision energy was set at 25 while the activation time was for $30 \mathrm{~ms}$. Xcalibur and Tune Plus software (Thermo Fisher Scientific, San Jose, CA) was used for data collection and analysis.

The experimental parameters described above were adopted from reference [20]. The authors strongly recommend further optimization when using a specific dication reagent for use in the detection of (a) specific anion(s). It is believed that these detection limits may be lowered when considerable time is given to optimization or when using a more sensitive mass spectrometer.

\section{Results and Discussion}

Table 1 provides the structure and mass of the wide variety of dications used in this study. Dications $\mathbf{I}-\mathbf{V}$ are phosphonium based while VI-XIV contain imidazolium structures ( $\mathbf{X}$ also contains a fluorocarbon linkage chain). Compounds XV-XXIII contain other charged moieties including trimethylammonium, pyridinium, and pyrrolidinium. In addition, some "mixed" and nonsymmetrical dicationic entities are included (XIX, $\mathbf{X X}, \mathbf{X X I}$, and XXIII).

Table 2 lists the limits of detection (LOD) for each of the six representative anions (benzenesulfonate, cyanate, perfluoro-octanoic acid, iodide, nitrate, monochloroacetic acid) when successfully paired with the 23 different dicationic reagents. These values were determined by direct injection ESI-MS (see the Experimental section) and are listed (from top to bottom) in order of sensitivity. Consequently, identifying the dicationic reagents that produce the best results (lowest LOD) as well as those which are ineffective is straight-forward (Table 2). The test anions were selected to provide a cross-section of ions having different sizes and functionalities [20]. Iodide, cyanate, and nitrate are relatively common and simple anions, but vary in size and number of constituent oxygen moieties. Benzenesulfonate (BZSN) was chosen as it is a somewhat larger organic anion and the only test analyte containing a sulfonate group. Monochloroacetic acid is a representative small halo-organic anion with environmental significance [24]. Perfluoro-octanoic acid (PFOA), a large, anionic fluorocarbon, is unlike any of the other anions. This, along with recent research interest in this compound as an environmental contaminant, makes it a good choice for inclusion in this study $[25,26]$.

It was expected that using different types of positively charged end groups would lead to differing performance. To show this effect, 10 different dicationic reagents that each contain the same pentane linkage can be compared. These 10 include dications II, VII, XIIXVII, and XIX-XX. Of these, four outperformed the rest. Both dications XIV and XVI produced good results (low LODs) even when compared with all other dications, while II and VII did almost as well. While both VII and XIV are imidazolium based compounds, II and XVI contain vastly different charged groups (phosphonium and pyrrolidinium). It must also be noted that XII produced the worst results of these 10 dications. Since XII is very close in structure and mass to XIII, it seems like the hydroxyl group leads to poorer detection limits. This is possibly due to its increased polarity, which would then lead to incomplete desolvation in the gas phase. It is of no surprise that BZSN paired better with the aromatic dications (other than XII), which points to 
Table 2. Absolute limits of detection for each anion detected as a dication-anion complex

\begin{tabular}{|c|c|c|c|c|c|}
\hline \multicolumn{2}{|c|}{ NCO- LOD } & \multicolumn{2}{|c|}{ PFOA- LOD } & \multicolumn{2}{|c|}{$\mathrm{NO}_{3}^{-} \mathrm{LOD}$} \\
\hline Dication & $\begin{array}{l}\text { Mass inj. } \\
\quad(\mathrm{ng})\end{array}$ & Dication & $\begin{array}{l}\text { Mass inj. } \\
\text { (ng) }\end{array}$ & Dication & $\begin{array}{l}\text { Mass inj. } \\
\text { (ng) }\end{array}$ \\
\hline $\mathrm{XVI}$ & $6.00 \mathrm{E}-02$ & VIII & $1.22 \mathrm{E}-04$ & VIII & 1.84E-03 \\
\hline XVIII & $8.00 \mathrm{E}-02$ & I & $2.50 \mathrm{E}-04$ & I & $5.00 \mathrm{E}-03$ \\
\hline$X X I$ & $2.00 \mathrm{E}-01$ & $X I$ & $5.00 \mathrm{E}-04$ & VII & $6.00 \mathrm{E}-03$ \\
\hline III & $3.00 \mathrm{E}-01$ & IV & 2.00E-03 & XVI & $1.60 \mathrm{E}-02$ \\
\hline IV & $6.00 \mathrm{E}-01$ & II & $3.00 \mathrm{E}-03$ & XIII & $2.00 \mathrm{E}-02$ \\
\hline II & $6.00 \mathrm{E}-01$ & V & 4.00E-03 & XVIII & $2.00 \mathrm{E}-02$ \\
\hline$x X$ & $8.00 \mathrm{E}-01$ & $x X$ & $4.00 \mathrm{E}-03$ & XIV & $2.00 \mathrm{E}-02$ \\
\hline XVII & $1.20 \mathrm{E}+00$ & XIV & $4.50 \mathrm{E}-03$ & XVII & $2.50 \mathrm{E}-02$ \\
\hline$X V$ & $3.00 \mathrm{E}+00$ & XVI & $6.00 \mathrm{E}-03$ & XII & $3.00 \mathrm{E}-02$ \\
\hline IX & $4.00 \mathrm{E}+00$ & XIX & 8.00E-03 & $\mathrm{XIX}$ & 4.00E-02 \\
\hline VIII & $6.42 \mathrm{E}+00$ & VII & 8.00E-03 & $\mathrm{IX}$ & 4.00E-02 \\
\hline$x$ & $8.00 \mathrm{E}+00$ & XVIII & $1.00 \mathrm{E}-02$ & III & $5.00 \mathrm{E}-02$ \\
\hline XXIII & $8.00 E+00$ & III & $1.00 \mathrm{E}-02$ & $x$ & $6.00 \mathrm{E}-02$ \\
\hline I & $1.50 \mathrm{E}+01$ & $x$ & 1.01E-02 & II & $6.50 \mathrm{E}-02$ \\
\hline XIX & $2.00 \mathrm{E}+01$ & VI & $1.40 \mathrm{E}-02$ & IV & $8.00 \mathrm{E}-02$ \\
\hline V & $2.00 \mathrm{E}+01$ & IX & $1.41 \mathrm{E}-02$ & $X X$ & $8.00 \mathrm{E}-02$ \\
\hline VI & $2.00 \mathrm{E}+01$ & XIII & 2.02E-02 & $X I$ & $1.20 \mathrm{E}-01$ \\
\hline VII & $2.00 \mathrm{E}+01$ & $X V$ & 2.01E-02 & V & $2.00 \mathrm{E}-01$ \\
\hline XIV & $1.50 \mathrm{E}+02$ & XVII & $5.00 \mathrm{E}-02$ & $X V$ & $2.00 \mathrm{E}-01$ \\
\hline XII & ND & XII & 6.06E-02 & VI & $6.00 \mathrm{E}-01$ \\
\hline XIII & ND & $X X I$ & $1.60 \mathrm{E}+00$ & XXIII & ND \\
\hline$X I$ & ND & XXIII & ND & XXI & ND \\
\hline XXII & ND & XXII & ND & XXII & ND \\
\hline \multicolumn{2}{|c|}{ BZSN $^{-}$LOD } & \multicolumn{2}{|c|}{ MCA- LOD } & \multicolumn{2}{|c|}{$I^{-}$LOD } \\
\hline Dication & Mass inj (ng) & Dication & Mass inj (ng) & Dication & Mass inj (ng) \\
\hline 1 & $1.03 \mathrm{E}-03$ & $\mathrm{XVI}$ & $6.00 \mathrm{E}-03$ & I & $1.08 \mathrm{E}-03$ \\
\hline XIV & $2.00 \mathrm{E}-03$ & II & $6.18 \mathrm{E}-03$ & $\mathrm{~V}$ & 1.62E-03 \\
\hline V & $2.06 \mathrm{E}-03$ & IV & $6.18 \mathrm{E}-03$ & XVI & $2.00 \mathrm{E}-03$ \\
\hline VIII & 2.06E-03 & XIV & $1.00 \mathrm{E}-02$ & IV & $2.16 \mathrm{E}-03$ \\
\hline$x$ & 4.04E-03 & III & 1.17E-02 & XIV & $4.00 \mathrm{E}-03$ \\
\hline VII & $5.00 \mathrm{E}-03$ & $x$ & $1.24 \mathrm{E}-02$ & XVIII & 4.04E-03 \\
\hline XIII & $5.00 \mathrm{E}-03$ & VIII & $1.50 \mathrm{E}-02$ & II & $4.32 \mathrm{E}-03$ \\
\hline IV & $6.18 \mathrm{E}-03$ & I & $1.65 \mathrm{E}-02$ & VIII & $6.00 \mathrm{E}-03$ \\
\hline IX & 7.00E-03 & VII & $1.80 \mathrm{E}-02$ & $X X$ & $6.48 \mathrm{E}-03$ \\
\hline VI & 8.00E-03 & XVII & 2.00E-02 & III & $6.48 \mathrm{E}-03$ \\
\hline$X V$ & 8.08E-03 & XIII & $2.00 \mathrm{E}-02$ & VII & 8.00E-03 \\
\hline XIX & $1.00 \mathrm{E}-02$ & $X X$ & 2.06E-02 & IX & 8.08E-03 \\
\hline III & $1.55 \mathrm{E}-02$ & XVIII & $3.00 \mathrm{E}-02$ & VI & $1.00 \mathrm{E}-02$ \\
\hline$x X$ & $1.55 \mathrm{E}-02$ & IX & $3.00 \mathrm{E}-02$ & XIII & $1.21 \mathrm{E}-02$ \\
\hline XII & $2.00 \mathrm{E}-02$ & $X V$ & 6.36E-02 & $X V I I$ & $2.00 \mathrm{E}-02$ \\
\hline XVI & $2.00 \mathrm{E}-02$ & XIX & $1.20 \mathrm{E}-01$ & $x$ & $2.00 \mathrm{E}-02$ \\
\hline II & $2.06 \mathrm{E}-02$ & $X I$ & $3.00 \mathrm{E}-01$ & $X I$ & $2.00 \mathrm{E}-02$ \\
\hline XVII & 4.00E-02 & XII & $5.00 \mathrm{E}-01$ & XII & 3.04E-02 \\
\hline XI & $5.00 \mathrm{E}-02$ & VI & $2.00 \mathrm{E}+01$ & XIX & $5.00 \mathrm{E}-02$ \\
\hline XVIII & $1.00 \mathrm{E}-01$ & XXI & $2.06 \mathrm{E}+01$ & $X V$ & $1.50 \mathrm{E}-01$ \\
\hline XXI & $4.00 \mathrm{E}+00$ & XXIII & $4.12 \mathrm{E}+01$ & XXIII & $4.32 \mathrm{E}+01$ \\
\hline XXII & ND & V & $5.16 \mathrm{E}+01$ & XXII & ND \\
\hline XXIII & ND & XXII & ND & $X X I$ & ND \\
\hline
\end{tabular}

ND: not detected (150 ng highest amount injected).

$\pi-\pi$ interactions playing a prominent role in gas-phase association. Interestingly, both iodide and cyanate do not seem to pair well with the imidazolium based dications.

The length of the "chain" connecting the cationic moieties is another parameter to consider. There are several analogous dications in this study that differ only by the length of the hydrocarbon linkage chain. Namely, I-III consist of phosphonium based dications, VI-VIII are all methyl-imidazolium based, and XVII and XVIII are alkyl-ammonium based. Looking at the phosphonium reagents, it can be seen that the C5 linked 
(II) and the C9 linked (III) behave similarly. However, the C3 linked (I) outperforms these with most of the anions tested, and by a wide margin. The only anions that are not improved upon are MCA (which have similar values) and cyanate. The opposite trend seems to be true for the methyl-imidazolium based reagents (VI-VIII), in that the larger C9 linked dication VIII produces superior results compared with all of the shorter linked imidazoliums for all anions. The two alkyl-ammonium dications behaved similarly, apart from PFOA and cyanate. For both of these anions, the C12 linked dication (XVIII) produced significantly lower detection limits. However, both the C3 linked phosphonium and the C9 linked imidazolium dications produced lower detection limits than did XVIII for all anions except for cyanate.

The effect of using different types of linkage chains was also studied. Three different chain types were studied. A p-xylene linker was used for dications $\mathbf{V}$ and IX, tetraethylene glycol was used for IV and XI, and a fluorocarbon chain is present on $\mathbf{X}$. In general, these more "exotic" linkage chain types were no better and generally worse than their corresponding optimal chain length hydrocarbon counterparts. Since the synthesis of these compounds is generally more complicated, there seems to be no advantage in using these linkage chain types.

A few dications studied did not fit into the categories above and thus, could not be compared in a systematic fashion. These compounds (XXI-XXIII) differ significantly from the others in that they do not contain two distinct charged moieties connected by a linkage chain. Some of these are naturally occurring compounds (XXI, XXIII) while one is a commercially available "diquat" (XXII). These types of compounds were not found to be useful for this method. Most of the anions could not even be detected as a complex with these particular dications. While it is unknown exactly how the dication interacts with the anion, it seems like an appropriate linkage chain that provides some flexibility is very important to ion association. This empirical observation may explain the poor performance of XXI-XXIII as well as why dications $\mathbf{V}$ and IX did not perform as well. The p-xylene linked dications (V, IX) are the most rigid amongst the symmetrical dications having a linkage chain. Clearly the flexibility of the dication is one factor that is important for complex formation. Ion mobility studies could provide insight into these dication-anion interactions and perhaps indicate how exactly the dication conforms to the anion [27, 28].

From the results described above, a few reagents stand out above the rest. The first is dication VIII. This dication performs well for all anions apart from cyanate. The best dication to analyze cyanate was found to be dication XVI, which also performs well for the other anions, especially iodide and nitrate. Dication $\mathbf{I}$ is also a reagent that should be among the first to be evaluated when using this method for any other anion, as it was the top performer for both benzenesulfonate and io- dide. Finally, while dication XIV was not the best for any particular anion, it generally was in the top quartile for all of the tested anions, and thus also is considered to be among the most useful dicationic reagents. These four dications (I, VIII, XIV, and XVI) encompass a phosphonium based dication, a pyrrolidinium based dication, and two imidazolium based dications. Each of these has a different optimum hydrocarbon linkage chain length. It is recommended that these four dications should be evaluated first when analyzing an anion that has not been previously studied with this gasphase ion association method.

It should be stated that the interpretation of the empirical results stated thus far have been primarily explained as a consequence of differing binding affinities between the dicationic reagent and the anion. However, it is essential to consider instrumental factors and the role they play in the sensitivity of these measurements. This is particularly true since only a single set of instrumental parameters was used for all dication-anion complex experiments. To demonstrate how instrumental response can significantly alter sensitivity, a complete optimization of instrumental parameters was done for the determination of monochloroacetate (MCA) using dication XVI. After optimization of both the electrospray and mass spectrometer parameters (see the Experimental section), the limit of detection was reduced by a factor of three (from $6.00 \mathrm{pg}$ to $2.00 \mathrm{pg}$, results not shown). It can clearly be seen that individual optimization will produce increased sensitivity for most of the anions in this study, and that instrument settings/configurations are important.

As an illustration of the pronounced effect of different dicationic reagents on the positive ion ESI-MS signal of anions, three analogous LC-ESI-MS analyses were compared (see Figure 1). Two of the recommended dications are used (VIII Figure $1 \mathrm{a}$ and XVI Figure 1b), as well as a moderately successful but not recommended dication XVII (Figure 1c). Each cation/anion complex was monitored at its appropriate $m / z$ (i.e., the sum of the mass of the dication and the mass of the anion). As can be seen, significant changes in peak area occur for each anion in successive chromatograms. As expected, the recommended dications (chromatograms A and B) outperform dication XVII. It should be noted that the worst performing dications (those in the bottom quartile of Table 2) would produce peaks that could not be discerned under the conditions of Figure 1. Also apparent in Figure 1 is that there are great differences even between the two recommended dications. So while the recommended dications generally perform well across the board, one should always be sure to test at least three or four of the reagents to obtain optimal signal intensity.

Often, this method can achieve significantly lower limits of detection by using tandem MS capabilities. Since this method takes place in the positive mode, the daughter fragment formed after excitation also must be a positive ion, which is a fragment of the 


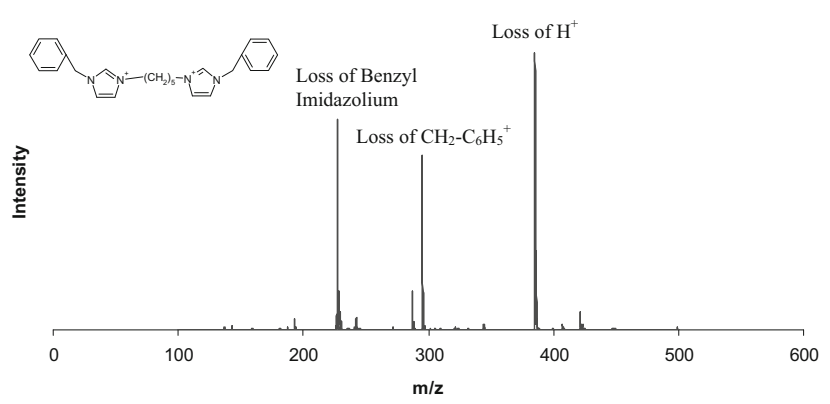

Figure 2. Mass spectrum of the mobile phase containing the dicationic reagent under typical operating settings for chromatography. Notice the three most prominent peaks are actually fragments of the dicationic reagent (XIV). These fragments can be monitored after excitation of a dication-anion complex to typically lower detection limits.

dication used. This is another key advantage of using this approach when determining the concentration of structurally-simpler anions (e.g., iodide) that cannot undergo fragmentation under MS/MS analysis in the negative ion mode. In a previous study, it was found that when a dication-anion complex was excited, it lost the anion and either a proton or a methylimidazolium group, resulting in a singly charged fragment that was left for detection. In many cases this reduced the LOD for a variety of anions. This is one distinct advantage of using the imidazolium-based dicationic reagents, as they lend themselves to MS/MS fragmentation more easily than other dications. A typical mass spectrum of the mobile phase under operating conditions (see the Experimental section) is shown in Figure 2. The dication used in this instance is compound XIV. Several discernable fragments can be seen in the background even without excitation. The main fragments include the peaks at 227.3 (loss of benzyl imidazolium), 295.3 (loss of $\left[\mathrm{CH}_{2}-\mathrm{C}_{6} \mathrm{H}_{5}\right]^{+}$), and 385.3 (loss of the acidic proton in the 2-position of imidazolium). Any of these peaks can be monitored after the excitation of the dication-anion complex, usually resulting in a significant increase in sensitivity. This increased sensitivity is illustrated in Figure 3 , which shows three separate chromatographic runs of $100 \mathrm{ng} / \mathrm{mL}$ of benzenesulfonate. While operating under negative ion mode with the addition of methanol post-column, a peak can be seen that gives a moderate $\mathrm{S} / \mathrm{N}$ of 14 . By simply using $40 \mu \mathrm{M}$ dication XIV in methanol and changing to the positive ion mode, an instant increase in the $\mathrm{S} / \mathrm{N}$ of almost 10 -fold (to 128) is seen. It can easily be seen why this approach is advantageous. This peak can be increased even further by the application of single reaction monitoring (SRM). When the transition of the complex mass $(\mathrm{m} / \mathrm{z}=543.3)$ to the fragment observed at 227.3 (loss of both the anion and benzyl imidazolium group) is monitored, the $\mathrm{S} / \mathrm{N}$ increases to 510. This is a 36-fold increase over using the "traditional" negative ion mode to monitor an anion. Since ESI is a "soft" ionization source, the relative abundance of fragments is surprisingly high. The amount of fragmentation seems to be dependent on the capillary temperature. A lower capillary temperature, while decreasing the amount of fragments, did not lead to an increase in sensitivity (possibly due to incomplete desolvation), while a higher capillary temperature $\left(>400{ }^{\circ} \mathrm{C}\right)$ actually led to decreased sensitivity. Interestingly, this fragmentation was only readily seen when using the imidazolium based dications, as other dications (such as phosphonium or pyrrolidinium types) did not lend themselves to significant fragmentation. Consequently, when using the non-imidazolium dications, no increases in sensitivity were seen when performing MS/MS. So while phosphonium or pyrrolidinium based dicationic reagents produce excellent results when using SIM, imidazolium based reagents should be evaluated if MS/MS capabilities are available. The four dications recommended above include two imidazolium based dications that can be used in MS/MS analysis.

\section{Conclusions}

The use of dicationic reagents to detect singly charged anions via gas-phase ion association has been shown to be a highly sensitive method and offers several significant improvements over using the negative ion mode when using tradition solvents. In this work, 23 different dications were evaluated to give insights as to the significant differences in dicationic reagents and which ones were most broadly useful. Four specific dicationic reagents (out of 23) stood out as far as producing superior performance and these are recommended when analyzing other anions. It was shown how this approach can be easily coupled to chromatography to study multiple anions. Also, the importance of choosing the correct dication to get significant signals for the anions of interest is demonstrated. Finally, the advan-

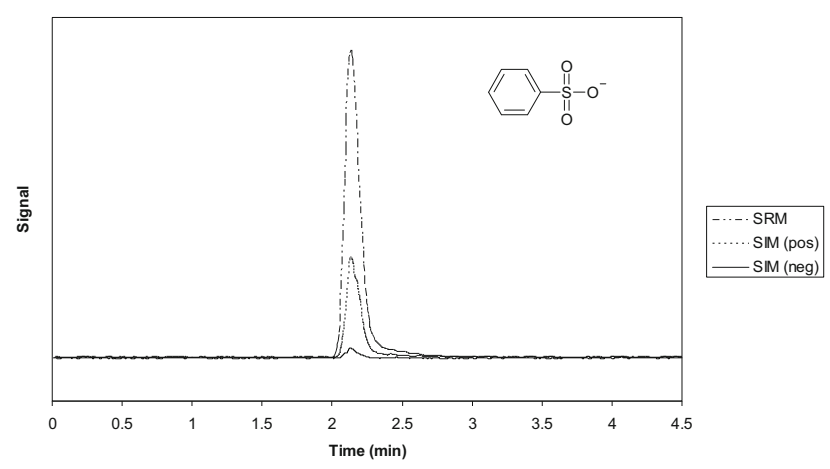

Figure 3. Overlapping chromatograms of three separate injections of a $100 \mathrm{ng} / \mathrm{mL}$ sample of benzene sulfonate. The solid line represents the use of negative mode, monitoring the mass of the anion (methanol being added post-column). When $40 \mu \mathrm{M}$ dication XIV in methanol is added post-column, the mass of the dicationanion complex can be monitored and gives a significant increase in S/N (dotted line). Finally, when single reaction monitoring is used, an even further increase in $\mathrm{S} / \mathrm{N}$ can be seen, as shown by the dashed line. 
tage of using the imidazolium based dications is shown through the application of MS/MS. Further work is needed to determine exactly how the dications interact with anions before any predictive capabilities are possible. Future work will include using this method to lower detection limits of methods that employ the negative ion mode and the possibility of studying doubly charged anions using tricationic species.

\section{Acknowledgments}

The authors thank Junmin Huang, Xinxin Han, and Aruna B. Wijeratne for synthesis of some of the dication compounds. The authors gratefully acknowledge the Robert A. Welch Foundation for its monetary support.

\section{References}

1. Pretsch, E. The New Wave of Ion - Selective Electrodes. Trends Anal. Chem. 2007, 26(1), 46-51.

2. Buck, R. P.; Lindner, E. Tracing the History of Selective Ion Sensors. Anal. Chem. 2001, 73(3), 88A-97A.

3. Haddad, P. R. Ion Chromatography. Anal. Bioanal. Chem. 2004, 379(3), 341-343.

4. Kuban, P.; Dasgupta, P. K. Capillary Ion Chromatography. J. Sep. Sci. 2004, 27(17/18), 1441-1457.

5. Hansen, E. H.; Wang, J.-H. The Three Generations of Flow Injection Analysis. Anal. Lett. 2004, 37(3), 345-359.

6. Miro, M.; Frenzel, W. What Flow Injection has to Offer in the Environmental Analytical Field. Microchim. Acta 2004, 148(1/2), 1-20.

7. Whitehouse, C. M.; Dreyer, R. N.; Yamashita, M.; Fenn, J. B. Electrospray Interface for Liquid Chromatographs and Mass Spectrometers. Anal. Chem. 1985, 57(3), 675-679.

8. Cech, N. B.; Enke, C. G. Practical Implications of Some Recent Studies in Electrospray Ionization Fundamentals. Mass Spectrom. Rev. 2001, 20(6), 362-387.

9. Voyksner, R. D. 1997; Combining Liquid Chromatography with Electrospray Mass Spectrometry. Cole, R. B., Ed.; In Electrospray Ionization Mass Spectrometry; pp 323-341.Wiley: New York,

10. Henriksen, T.; Juhler, R. K.; Svensmark, B.; Cech, N. B. The Relative Influences of Acidity and Polarity on Responsiveness of Small Organic Molecules to Analysis with Negative Ion Electrospray Ionization Mass Spectrometry (ESI-MS). J. Am. Soc. Mass Spectrom. 2005, 16(4), 446-455.

11. Kebarle, P.; Yeunghaw, H. 1997; On the Mechanism of Electrospray Mass Spectrometry. Cole, R. B., Ed.; In Electrospray Ionization Mass Spectrometry; p 14Wiley: New York,
12. Cole, R. B.; Zhu, J.-H. Chloride Anion Attachment in Negative Ion Electrospray Ionization Mass Spectrometry. Rapid Commun. Mass Spectrom. 1999, 13(7), 607-611.

13. Apffel, A.; Chakel, J. A.; Fischer, S.; Lichtenwalter, K.; Hancock, W. S. Analysis of Oligonucleotides by HPLC-Electrospray Ionization Mass Spectrometry. Anal. Chem. 1997, 69(7), 1320-1325.

14. Straub, R. F.; Voyksner, R. D. Negative Ion Formation in Electrospray Mass Spectrometry. J. Am. Soc. Mass Spectrom. 1993, 4(7), 578-587.

15. Cole, R. B.; Harrata, A. K. Charge-State Distribution and ElectricDischarge Suppression in Negative-Ion Electrospray Mass Spectrometry Using Chlorinated Solvents. Rapid Commun. Mass Spectrom. 1992, 6(8), 536-539.

16. Martinelango, P. K.; Anderson, J. L.; Dasgupta, P. K.; Armstrong, D. W.; Al-Horr, R. S.; Slingsby, R. W. Gas-Phase Ion Association Provides Increased Selectivity and Sensitivity for Measuring Perchlorate by Mass Spectrometry. Anal. Chem. 2005, 77(15), 4829-4835.

17. Martinelango, P. K; Guemues, G.; Dasgupta, P. K. Matrix Interference Free Determination of Perchlorate in Urine by Ion Association-Ion Chromatography-Mass Spectrometry. Anal. Chim. Acta 2006, 567(1), 79-86.

18. Dyke, J. V.; Kirk, A. B.; Kalyani, M., P.; Dasgupta, P. K. Sample Processing Method for the Determination of Perchlorate in Milk. Anal. Chim. Acta 2006, 567(1), 73-78.

19. Martinelango, P. K.; Tian, K.; Dasgupta, P. K. Perchlorate in Seawater. Anal. Chim. Acta 2006, 567(1), 100-107.

20. Soukup-Hein, R. J.; Remsburg, J. W.; Dasgupta, P. K.; Amstrong, D. W. A General, Sensitive, Positive Ion Mode ESI-MS Approach for the Analysis of Singly Charged Inorganic and Organic Anions Using a Dicationic Reagent. Anal. Chem. 2007, 79(19), 7346-7352.

21. Anderson, J. L.; Ding, R.; Ellern, A.; Armstrong, D. W. Structure and Properties of High Stability Geminal Dicationic Ionic Liquids. J. Am. Chem. Soc. 2005, 127(2), 593-604.

22. Huang, K.; Han, X.; Zhang, X.; Armstrong, D. W. PEG-linked Geminal Dicationic Ionic Liquids as Selective, High Stability Gas Chromatographic Stationary Phases. Anal. Bioanal. Chem. 2007, in press.

23. Payagala, T.; Huang, J.; Breitbach, Z. S.; Sharma, P. S.; Armstrong, D. W Unsymmetrical Dicationic Ionic Liquids: Manipulation of Physicochemical Properties Using Specific Structural Architectures. Chem. Mater. J. 2007, 19(24), 5848-5850.

24. Giller, S.; Le Curieux, F.; Erb, F.; Marzin, D. Comparative Genotoxicity of Halogenated Acetic Acids Found in Drinking Water. Mutagenesis 1997, 12(5), 321-328.

25. Kennedy, G. L., Jr.; Butenhoff, J. L.; Olsen, G. W.; O'Connor, J. C.; Seacat, A. M.; Perkins, R. G.; Biegel, L. B.; Murphy, S R.; Farrar, D. G. The Toxicology of Perfluoro-octanoate. Crit. Rev. Toxicol. 2004, 34(4), 351384.

26. Kumar, K. S. Fluorinated Organic Chemicals: A Review. Res. J. Chem. Environ. 2005, 9(3), 50-79.

27. Borsdorf, H.; Eiceman, G. A. Ion Mobility Spectrometry: Principles and Applications. Appl. Spectrosc. Rev. 2006, 41(4), 323-375.

28. Wyttenbach, T.; Bowers, M. T. Gas-Phase Conformations: The Ion Mobility/Ion Chromatography Method. Topics Curr. Chem. 2003, 225 (Mod. Mass Spectrom.) 207-232. 